LA- - 10921

DE87 010829

\title{
Plutonium Process Control \\ Using an Advanced On-Line Gamma Monitor for Uranium, Plutonium, and Americium
}

\author{
S. Fredric Marsh \\ Michael C. Miller
}

\section{DISCLAIMER}

\begin{abstract}
This report was prepared as an account of work sponsored by an agency of the United States Government. Neither the United States Government nor any agency thereof, nor any of their employees, makes any warranty, express or implied, or assumes any legal liability or responsibility for the accuracy, completeness, or usefulness of any information, apparatus, product, or process disclosed, or represents that its use would not infringe privately owned rights. Reference herein to any specific commercial product, process, or service by trade name, trademark, manufacturer, or otherwise does not necessarily constitute or imply its endorsement, recommendation, or favoring by the United States Government or any agency therwof. The views and opinions of authors expressed herein do not necessarily state or reflect those of the United States Government or any ayency thereof.
\end{abstract}

\section{MASTIIII}
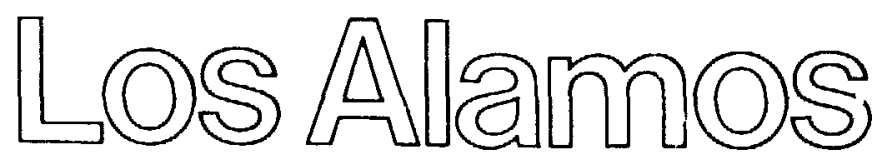

Los Alamos National Laboratory Los Alamos,New Mexico 87545 


\title{
PLUTONIUM PROCESS CONTROL USING AIN ADVANCED ON-LINE GAMMA MONITOR FOR URANIUM, PLUTONIUM, AND AMERICIUM
}

\author{
by
}

\author{
S. Fredric Marsh and Michael C. Miller
}

\begin{abstract}
An on-line gamma monitor has been developed to profile uranium, piutonium, and americium in waste and product streams of the anion exchange process used to recover and purify plutonium at the Los Alamos Plutonium Facility. The gamma monitor employs passive gamma spectrometry to measure ${ }^{241} \mathrm{Am}$ and ${ }^{239} \mathrm{Pu}$, based on their $59.5-\mathrm{keV}$ and 129-keV gamma rays, respectively. Because natural and depleted uranium present in typical process streams have no gamma rays suitable for measurement by such passive methods, uranium measurement requires a novel and less direct technique. Plutonium-241, which is always present in plutonium processed at Los Alamos, decays primarily by beta emission to form ${ }^{241} \mathrm{Am}$. However, a small fraction of ${ }^{241} \mathrm{Pu}$ decays by alpha emission to 6.8-day ${ }^{237} \mathrm{U}$. The short half-life and 208-keV gamma energy of ${ }^{237} U$ make it an ideal radiotracer to mark the position of macro amounts of uranium impurity in the separation process. The real-time data obtained from an operating process allow operators to optimize many process parameters. The gamma monitor also provides a permanent record of the daily performance of each ion exchange system.
\end{abstract}

\section{INTRODUCTION}

The major aqueous process used to recover and purify plutonium at the Los Alamos Plutonium Facility (TA-55) is anion exchange in nitric acid. This process is especially well suited for purifying plutonium, as $\mathrm{Pu}(\mathrm{IV})$ is more strongly sorbed than any other ion, and few other metal ions show even moderate sorption from nitric acid, as seen in Fig. 1.

The anion exchange process is outlined in schematic form in Fig. 2. Major steps of the process include (1) adjusting the plutonium oxidation state to form the strongly sorbed $\mathrm{Pu}(\mathrm{IV})$ nitrato complex, (2) adjusting other parameters such as acid concentration to maximize the retention of plutonium while minimizing the retention of impurity elements, (3) sorbing the anionic nitrato complex of $\mathrm{Pu}(\mathrm{IV})$ on the anion exchange column, (4) eluting impurities with nitric acid wash, and finally (5) eluting plutonium after reduction of $\mathrm{Pu}(\mathrm{IV})$ to $\mathrm{Pu}(\mathrm{III})$ with hydroxylammonium nitrate. The chemical treatment procedure is detailed in a separate report. ${ }^{1}$

Impurity elements to be separated typically include alkali metals, alkaline earths, transition metals, aluminum, americium, and uranium. Most of these impurity elements are, at best, weakly sorbed from nitric acid. Uranium, a common impurity, is sufficiently 

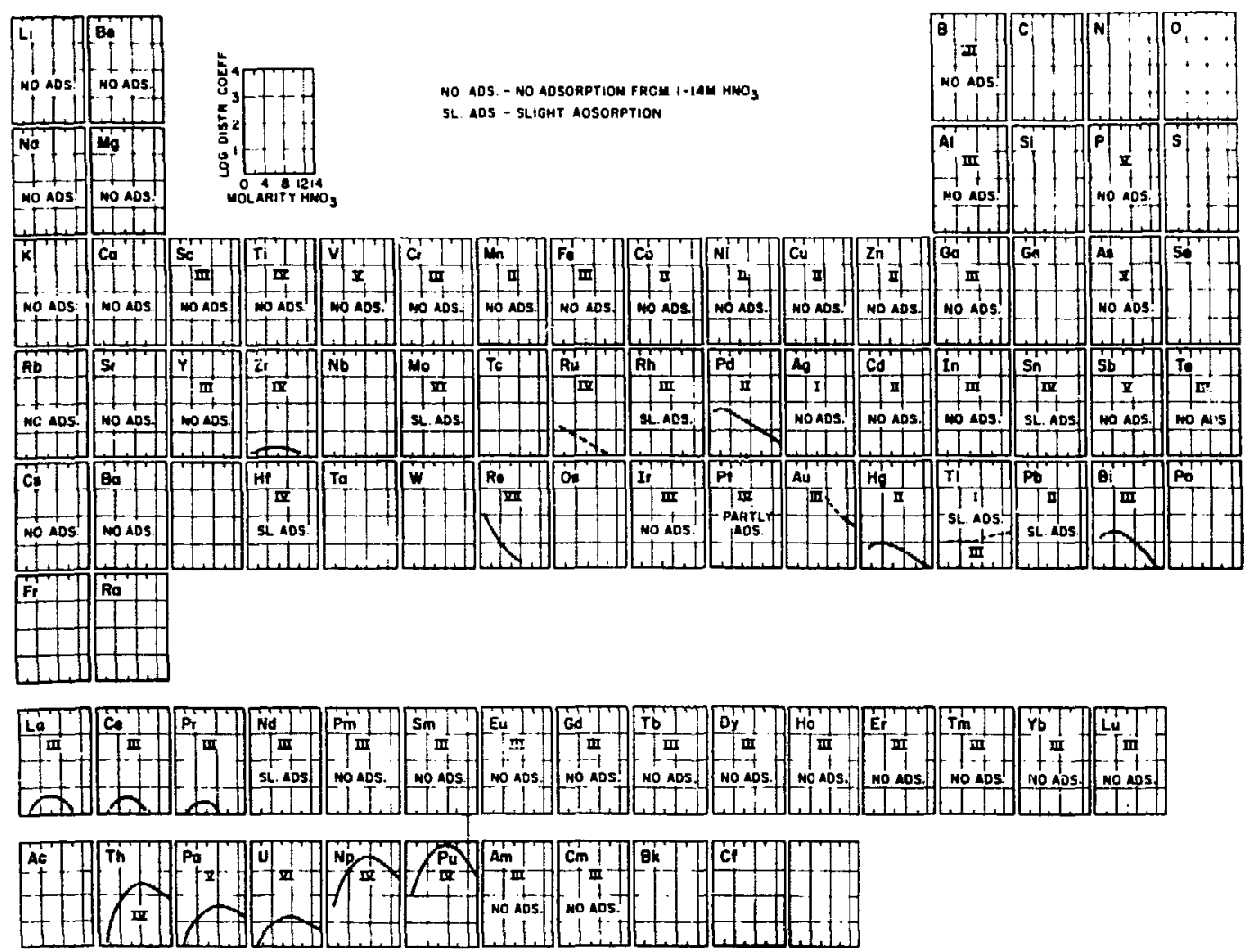

Fig. 1. Distribution of elements from nitric acid onto strong-base anion exchange resin.

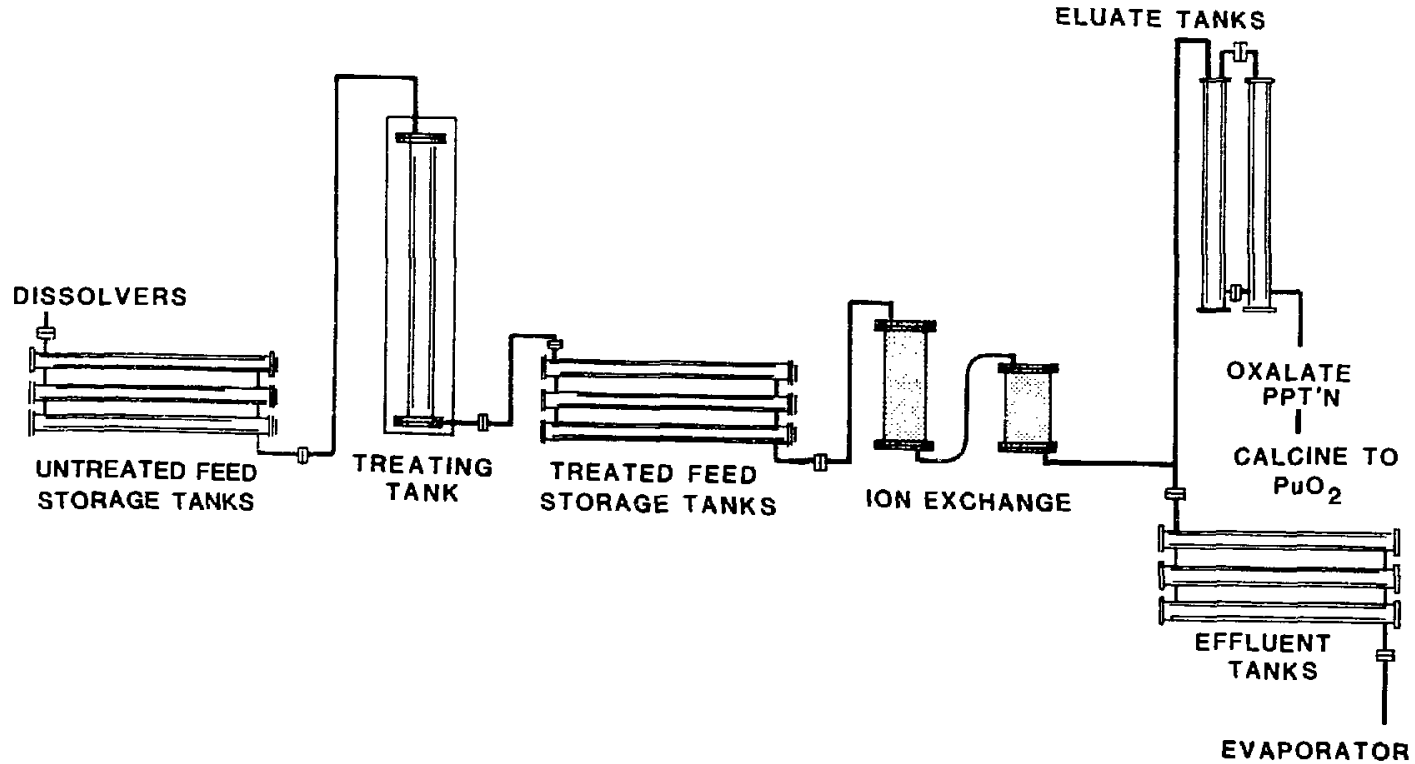

Fig. 2. Schematic of the anion exchange process used at Los Alamos. 
sorbed in this system to require a larger-than-usual volume of ritric acid wash solution to completely remove it from the anion exchange column.

The Los Alamos anion exchange process has traditionally been operated according to a fixed procedure, even though feed solutions typically vary over a wide range of impurity compositions and concentrations. In the past, process operators estimated the volume of acid wash solution needed to remove impurities on the basis of visual observations and intuition alone. This technique frequently meant that operators used (1) too little acid wash, which resulted in a plutonium product that contained excessive levels of impurities, or (2) too much acid wash, which generated unn:cessarily large volumes of contaminated acid wash that was time consuming and expensive to process.

The objective of this work was to develop an online measurement system that provides process operators with the real-time information they need to accurately determine the amount of wash solution needed to remove all impurities. This initial objective was expanded to include real-time status information related to other phases of the anion exchange process operation.

\section{INSTRUMENTS}

Germanium Gamma Detector. High-purity, coaxial detector with FWHM of $\approx 1.9 \mathrm{keV}$ at 1.33 $\mathrm{MeV}$ and $\approx 10 \%$ efficiency, with streamline cryostat, horizontal-detector configuration, and cooled FET. EG\&G ORTEC, Oak Ridge, Tennessee.

Multichannel Analyzer. Aptec SA-1 analyzer with 2048 memory channels and data output in digital or analog form. System features "Real-Time Gamma Analysis" mode in which user-defined algorithms periodically process acquired data. Aptec Nuclear, Inc., Lewiston, New York.

Analog-to-Digital Converter. Canberra model 8075 with 8192 channel conversion gain and range, single-width NIM, 100-MHz clock rate. Canberra Industries, Meriden, Connecticut.

Digital Stabilizer. Canberra model 8232, simultaneous zero-point and gain stabilization, single-width NIM, 14-bit ADC input. Canberra Industries, Meriden, Connecticut.
Spectroscopy Amplifier. ORTEC model 572, with baseline restorer and pile-up rejection. EG\&G ORTEC, Oak Ridge, Tennessee.

Timer/Counter. ORTEC model 871. EG\&G ORTEC, Oak Ridge, Tennessee.

Bias Supply. Canberra model 3105 high-voltage power supply, single-width NIM, 0 to $\pm 5 \mathrm{kV}$. Canberra Industries, Meriden, Connecticut.

Minibin. ORTEC model 4001M with power supply, 6-slot capacity. EG\&G ORTEC, Oak Ridge, Tennessee.

Stripchart Recorder. Servocorder Dx model SR6335, vertical servo-type, three independent recording channels, $10 \mathrm{mV}$ to $100 \mathrm{~V}$ input, multispeed chart output. Western Graphtec Inc., Irvine, California.

\section{EXPERIMENTAL}

The behavior of plutonium and americium is of special interest in the anion exchange process, because plutonium is the most strongly sorbed element, and arnericium is one of the nonsorbed elements. Thus, the behavior of these two actinides encompasses the behavior of all other elements in the process. We monitor these two elements by measuring the gamma rays of ${ }^{239} \mathrm{Pu}$ and ${ }^{241} \mathrm{Am}$ at $129 \mathrm{keV}$ and $59.5 \mathrm{keV}$, respectively.

The behavior of uranium in a plutonium purification process also is of special interest because it usually is present at levels that exceed allowable impurity specifications. The magnitude of uranium retention on anion exchange resin from nitric acid is between those of plutonium and americium. Uranium is weakly enough sorbed to allow its complete removal by adequate nitric acid washing, but it is strongly enough sorbed that most other impurity elements elute first. Uranium therefore can serve as an internal indicator to confirm the complete removal of numerous other impurity elements that precede it. Confirmation that uranium removal is complete marks the complete removal of most other impurity elements as well, and serves as a reliable signal to the operator that washing can be stopped and elution begun.

Because natural or depleted uranium impurity typically found in Los Alamos feed materials has no passive gamma rays suitable for direct, measurement, we 
devised a novel and indirect technique for monitoring this element. Plutonium processed in our facility always contains ${ }^{241} \mathrm{Pu}$. Plutonium-241 primarily decays by beta emission to ${ }^{241} \mathrm{Am}$; however, a very small fraction decays by alpha emission to 6.8-day ${ }^{237} \mathrm{U}$ (Fig. 3). Although this minor alpha branch produces relatively few atoms of ${ }^{237} \mathrm{U}$, the high specific activity and abundant $208-\mathrm{keV}$ gamma ray of ${ }^{237} \mathrm{U}$ make it an ideal radiotracer to monitor the behavior of all uranium impurity. (Americium-241 has a minor alpha branch of less than $10^{-2} \%$ that also decays through the $208-\mathrm{keV}$ excited state of ${ }^{237} \mathrm{~Np}$. However, by using its known ratio to the $59.5-\mathrm{keV}$ gamma ray of ${ }^{241} \mathrm{Am}$, this small contribution can easily be removed.)

It must be emphasized that the quantity of ${ }^{237} \mathrm{U}$ present is in no way related to the absolute amount of uranium present; it is a function only of the quantity of ${ }^{241} \mathrm{Pu}$ parent present and the elapised time since previous separation. However, the ${ }^{237} \mathrm{U}$, which is chemically

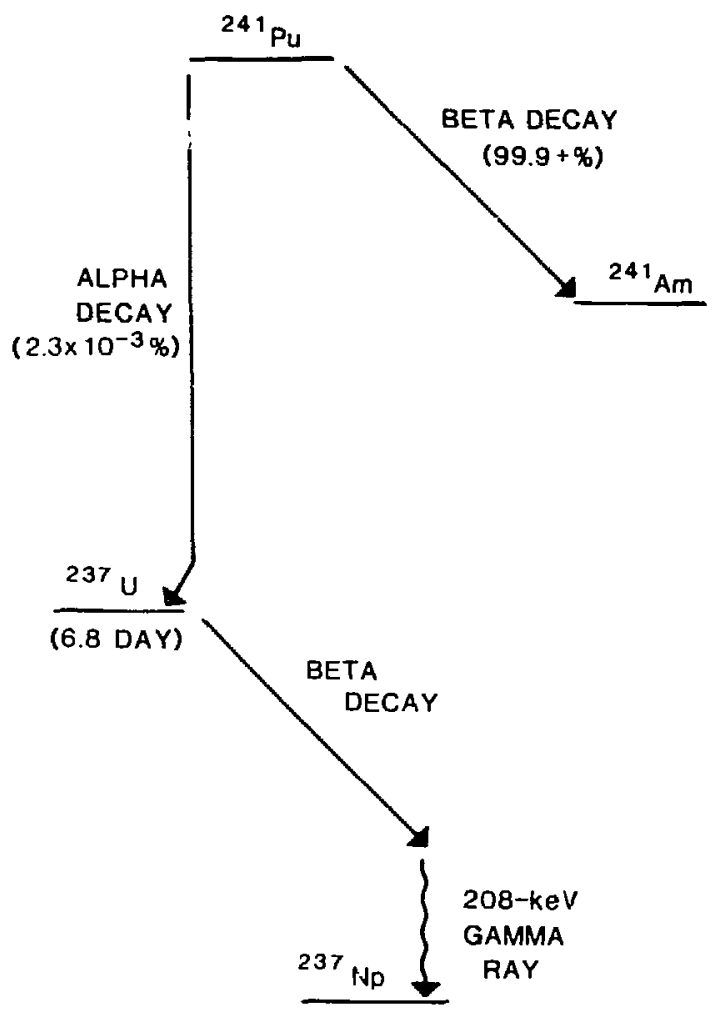

Flg. 3. Formation of ${ }^{237} \mathrm{U}$ by a minor alpha-decay branch of ${ }^{241} \mathrm{Pu}$. indistinguishable from othrr !ranium present, traces the relative concentration profile of all uranium impurity as it passes through the separation process.

The initial prototype instrument ${ }^{2}$ consisted of a sodium iodide detector, an amplifier, and three singlechannel analyzers that provided analog signals to a three-pen stripchart recorder (Fig. 4). This unsophisticated, rninimum-cost version was suficient to provide proof-of-principle data. Based on the success of the prototype instrument, the second-generation instrument described in this report was designed and built.

The second-generation on-line gamma monitor consists of a high-purity germanium detector, a highresolution spectroscopy amplifier, and a particularly versatile multichannel analyzer. Supporting instrumentation includes a digital zero/gain stabilizer and ratemeter, as shown in Fig. 5. Zero/gain stabilization is obtained by continuously monitoring a $0.1-\mu \mathrm{ci}$ ${ }^{133} \mathrm{Ba}$ source attached to the detector. The zero-point correction is determined from the position of the 80$\mathrm{keV}$ peak, whereas the gain correction is based on the position of the $356-\mathrm{keV}$ peak.

The selected Aptec multichannel analyzer (MCA) incorporates certain unique features that make it especially well suited for monitoring several fluctuating radioactive sources as a function of time. Regions of interest (ROI) are first entered to define selected energy windows. As many as 20 user-defined equations may be applied to convert the raw spectral data into convenient units, such as disintegration rate or concentration of the nuclide of interest. Execution of this "Real-Time Gamma Analysis" feature of the Aptec MCA immediately follows the preset data collection period. The resulting data are output as analog or digital signals, as well as through the video display.

In our case, the data collection period was $30 \mathrm{sec}-$ onds (livetime). ROIs were centered on $59.5 \mathrm{keV}$ for ${ }^{241} \mathrm{Am}, 129 \mathrm{keV}$ for ${ }^{239} \mathrm{Pu}$, and $208 \mathrm{keV}$ for ${ }^{237} \mathrm{U}$. Logarithms of the net peak rates were calculated for these three ROIs, and analog signals proportional to their values were routed to a three-pen stripchart recorder.

We selected logarithmic stripchart output to expand the representation of low values, while compressing that of high values. This allowed presentation of a very wide dynamic range, while high sensitivity was retained for the low values where it is most needed. Different-color pens on the stripchart recorder allowed the process status of each actinide element to be quickly and easily ascertained. 


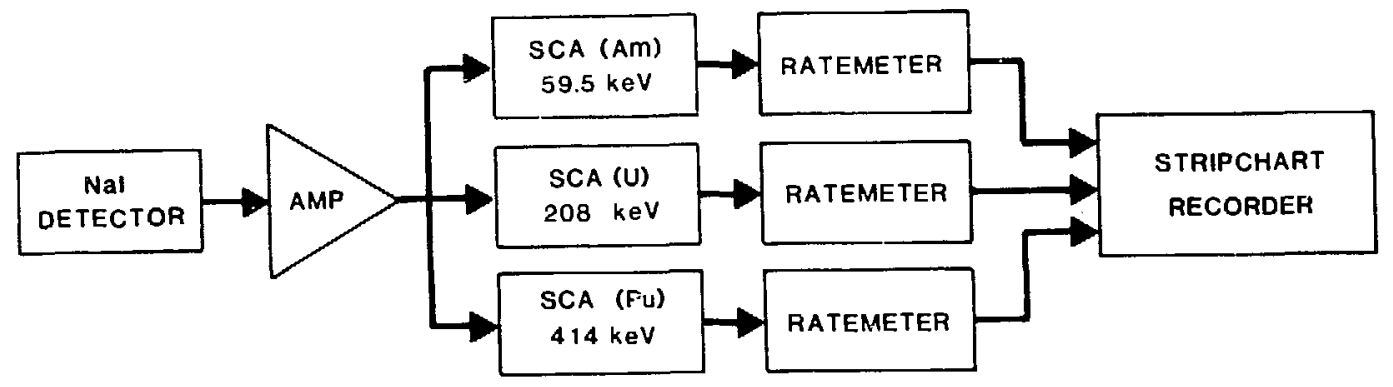

Fig. 4. Schematic of prototype on-line gamma monitor.

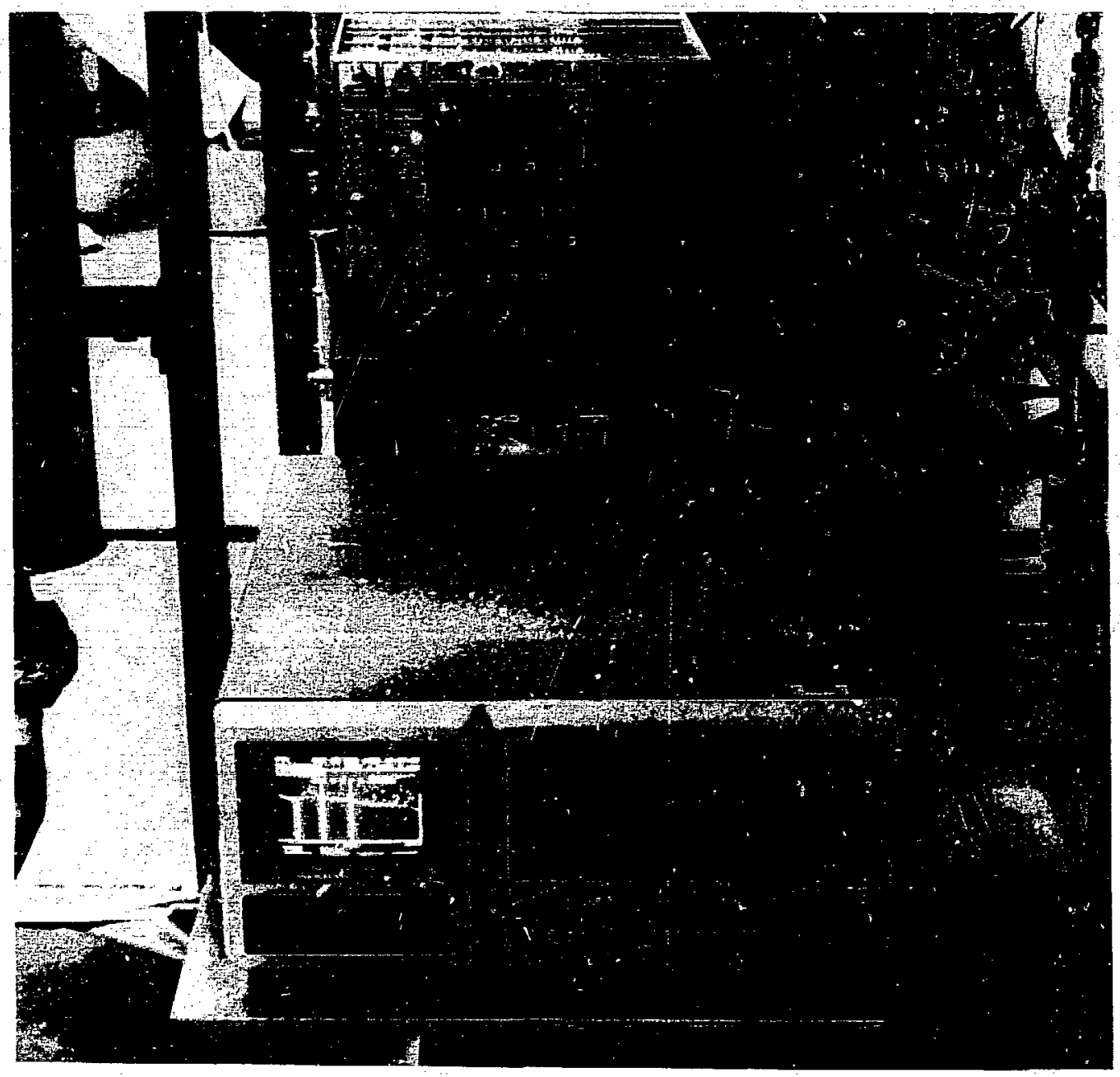

Flg. 5. Multichannel analyzer-based on-line gamma monitor. 


\section{RESULTS AND DISCUSSION}

\section{Process Control}

The on-line gamma monitor assays the outlet stream of the anion exchange column (Fig. 2) before it is diverted to either the effluent or eluate storage. Based on the distribution coefficients shown in Fig. 1, one would expect $A \mathrm{~m}(\mathrm{lII})$, which does not form an anionic complex in nitric acid, to elute first from the anion exchange column. Uranium (VI), which forms a weakly sorbed anionic complex, should elute next. And $\mathrm{Pu}(\mathrm{IV})$, which forms a strongly sorbed anionic complex, should remain on the anion exchange column until it is reduced to nonsorbed $\mathrm{Pu}$ (III).

A typical stripchart record (Fig. 6) shows the expected anion exchange behavior for these three actinide elements. Americium appears in the effuent as soon as the feed solution front reaches the column exit. Americium quickly reaches a constant value that signifies equal concentrations in the column inlet and outlet streams. Uranium is slower to appear in the effluent stream, as its weak sorption is sufficient to delay its exit from the column. In a properly operating ion exchange process, plutonium as $\mathrm{Pu}(\mathrm{IV})$ remains low and constant until it is chemically converted to norisorbed $\mathrm{Pu}(\mathrm{III})$, which elutes.

The real-time process status information illustrated in Fig. 6 allows the operator to decide when the acid wash volume has been sufficient to remove major impurities, as indicated by the complete removal of uranium, and when to start and stop collection of plutonium product during its elution. Before the installation of this monitor, process operators had no guidance for these decisions other than visual observations. The on-line monitor thus serves a vital and previously unmet process control function.

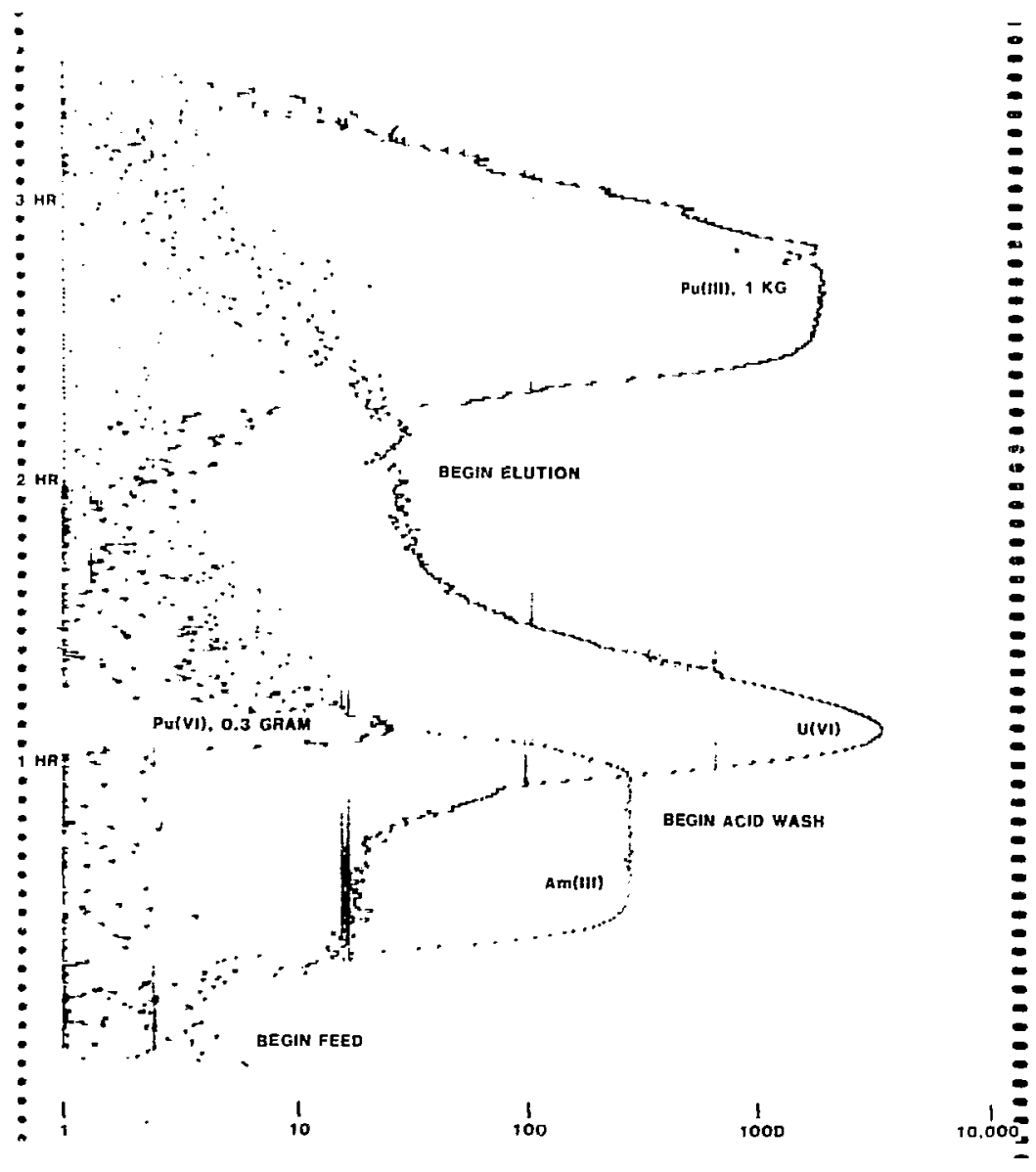

Fig. 6. Typical stripchart record of anion exchange separation process. 


\section{Process Diagnostics}

In addition to its application to process control, the on-line monitor also serves as a powerful diagnostic tool. This use is illustrated in Fig. 7, a stripchart record of the effluent stream from another feed solution in which an early plutonium peak appears near the uranium impurity peak. Because the plutonium peak is so well defined, it is unlikely that plutonium breakthrough accounts for it. The fact that the plutonium peak nearly coincides with the uranium peak led us to suspect that the peak represented $\mathrm{Pu}$ (VI), whose anion exchange behavior is very similar to that of U(VI). Portions of effluent collected at the suspect peak posiiion were analyzed by spectrophotometry, as shown in: Fig. 8. The characteristic absorbance peak at $831 \mathrm{~nm}$ confirms that only $\mathrm{Pu}(\mathrm{VI})$ is present.

Based on these data, a modified treatment ${ }^{1}$ of the feed solution was devised and tested on another portion of identical feed solution. The success of the modifed treatment is confirmed by Fig. 9; no sign of the previous $\mathrm{Pu}(\mathrm{VI})$ peak is now seen. Syectrophotometric analysis of the effluent solution verified the absence of $\mathrm{Pu}(\mathrm{VI})$, as shown in Fig. 10.

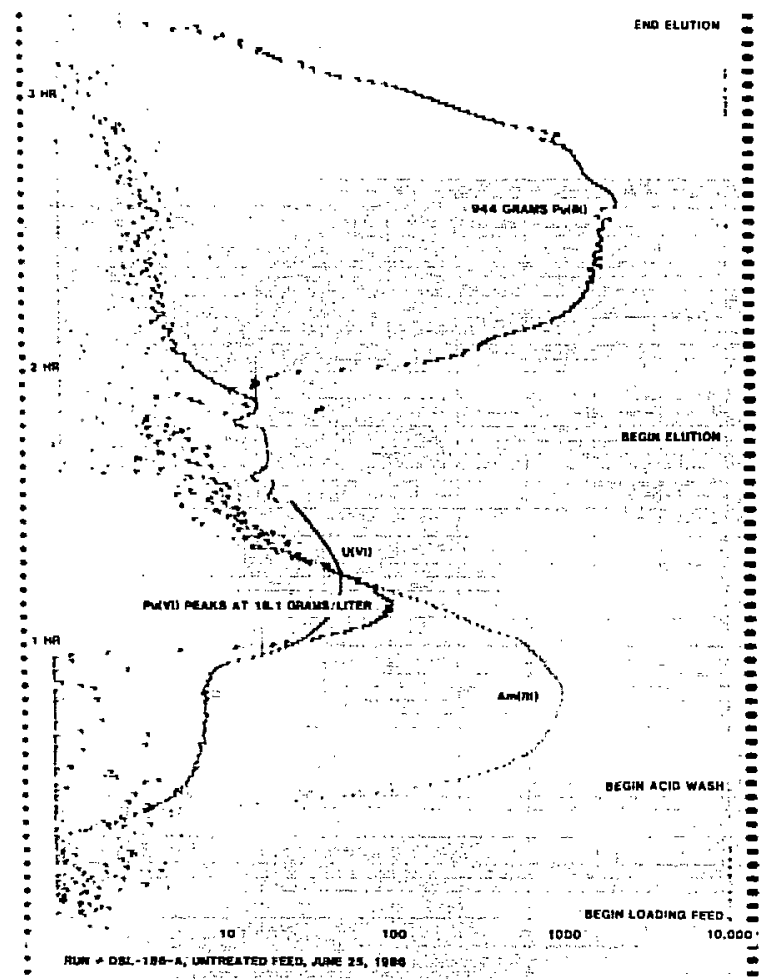

Fig. 7. Stripchart recrird of untreated feed solution. (Note the peak that indicates $\mathrm{Pu}$ (VI) being lost to the effluent waste stream.)

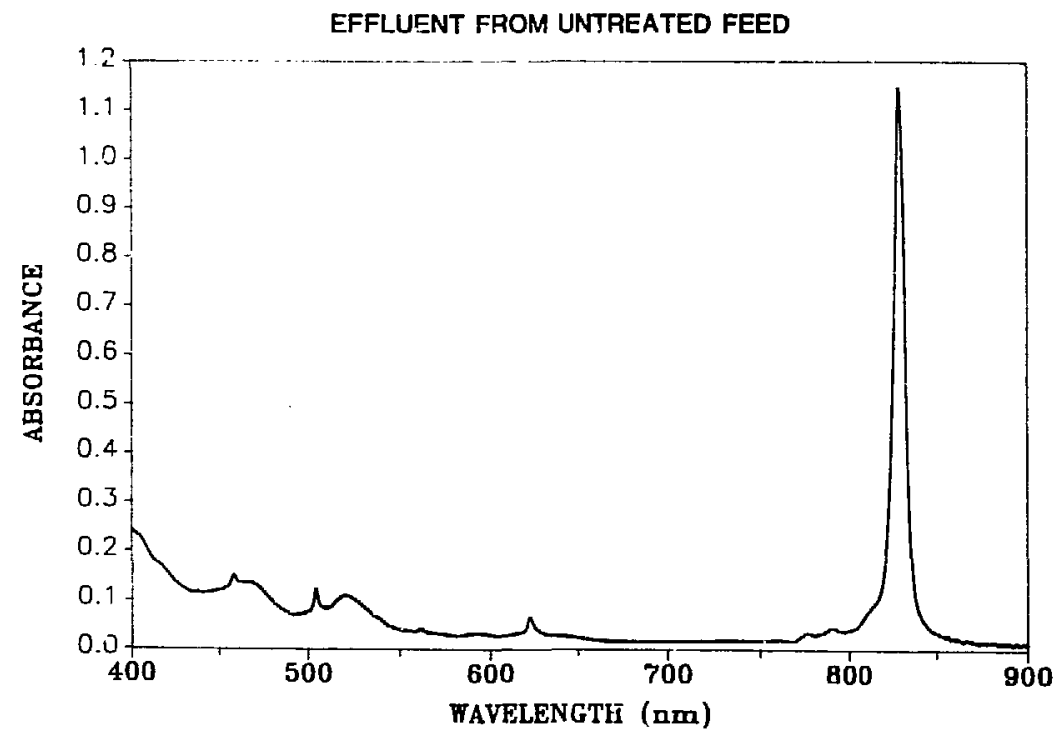

FIg. 8. Absorbance spectrum confirming the presence of $\mathrm{Pu}(\mathrm{VI})$ in untreated effluent solution. 


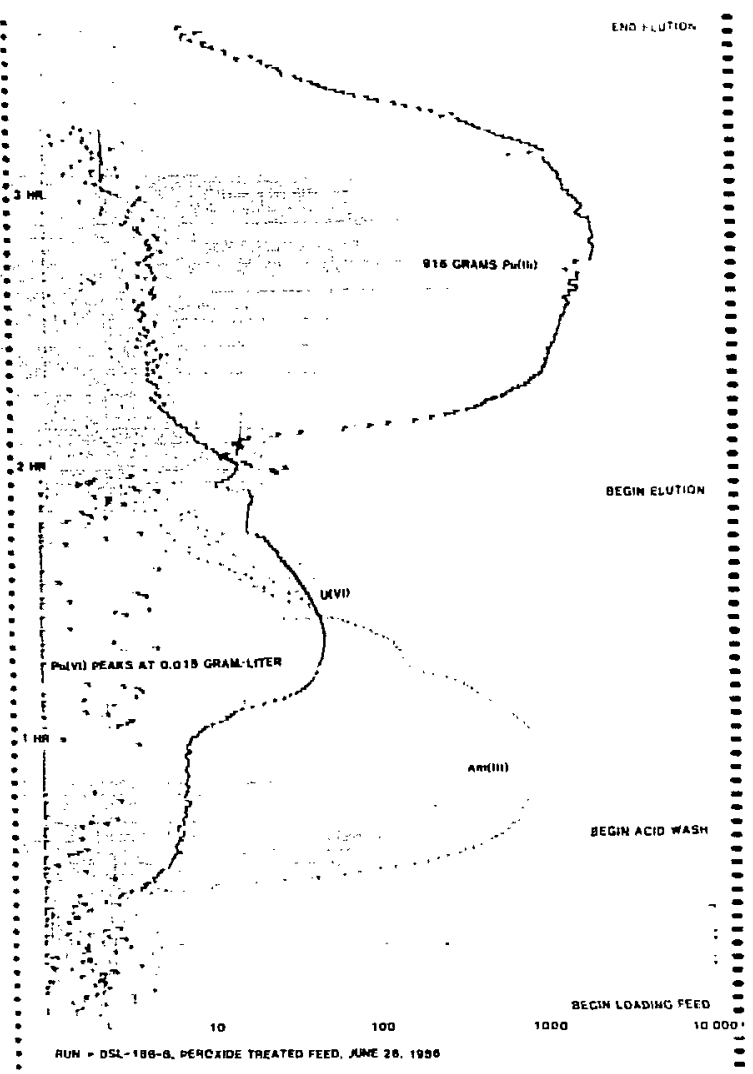

Fig. 3. Stripchart record of peroxide-treated feed solution. (Note the absence of the $\mathrm{Pu}(\mathrm{VI})$ peak after peroxide treatment.)

\section{Application to Semiquantitative Assay}

The on-line gamma monitor was designed to provide data needed (1) initially for manual process control and (2) eventually for automated process control. This objective, which requires only relative and trend information from an operating process, has been met. In addition, we have considered how this system might provide semiquantitative measurement of plutonium in the product stream. In principle, this can be done. In practice, however, two minor problems and one major problem must first be overcome.

One problem is that hydroxylammonium nitrate, used to chemically reduce $\mathrm{Pu}(\mathrm{IV})$ to $\mathrm{Pu}$ (III), produces nitrogen gas as a reaction product. Thus, the eluate stream being monitored contains a mixture of liquid and gas. For our application, this inhomogeneity presents an insurmountable obstacle to reliable assay measurements; we are therefore investigating mechanical means of eliminating the gas component of this eluate stream to provide to the on-line gamma monitor a gas-free liquid stream, which is an essential prerequisite of even semiquantitative assay.

A second problem involves attenuation of the gamma rays being assayed. The major portion of this attenuation is constant, as it arises from stationary $\mathbf{a b}$ sorbing materials that separate the detector from the plutonium solution being measured. A second attenuation component arises from absorption of the gamma

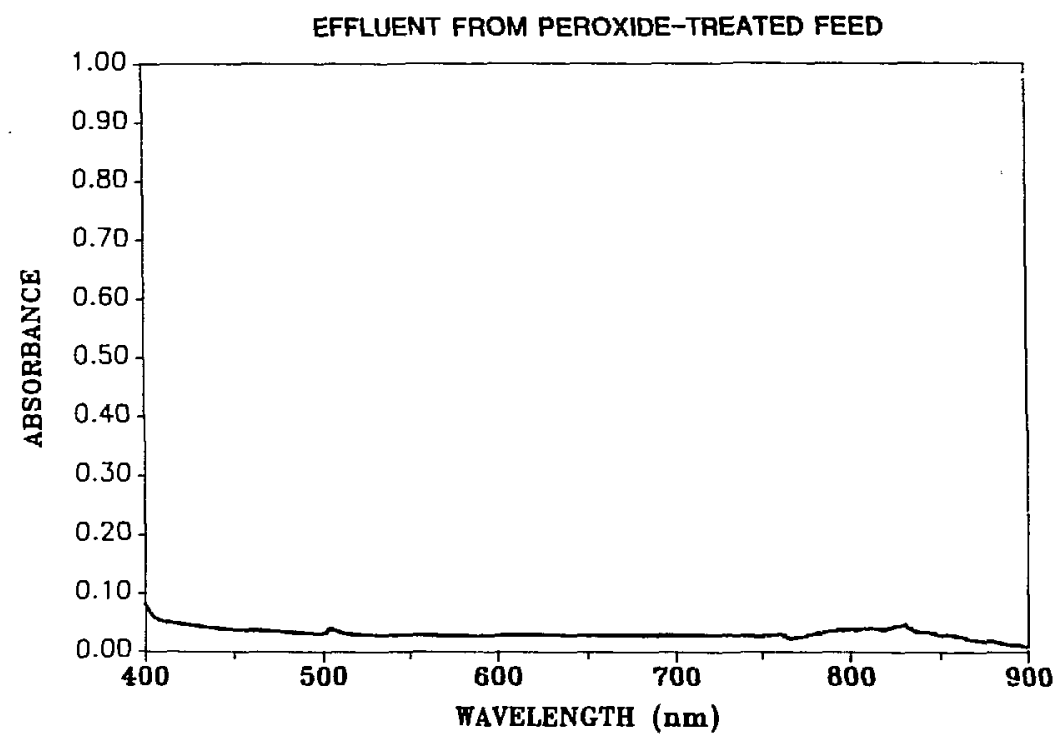

Fig. 10. Absorbance spectrum confirming the absence of $\mathrm{Pu}(\mathrm{VI})$ in peroxide-treated effluent solution. 
rays within the plutonium solution itself, and this component is known to vary with the concentration of plutonium in the solution being measured.

One method of correcting for self-attenuation within the solution is to use a transmission source. ${ }^{3}$ The detector in this case measures not only gamma rays emitted from nuclides within the solution, but also the intensity of a suitable gamma ray after its passage through the solution being assayed. From this transmitted intensity, we may determine the linear attenuation coefficient of the solution. Data reduction, in this case, incorporates a correction factor based on the determined attenuation coefficient. This approach, known to be quite accurate, requires the use of a small computer in addition to the MCA. Another approach, known as the differential attenuation technique, has the advantage of requiring no additional system hardware components. The differential attenuation method ${ }^{4}$ uses the ratio of two gamma rays emitted from a single nuclide to provide information related to attenuation within the solution. Because the linear attenuation cuefficient is a function of both gamma energy and the attenuating solution composition, gamma rays of different energies will be attenuated to different extents. Plutonium-239 emits gamma rays of 129 $\mathrm{keV}$ and $414 \mathrm{keV}$, both of which are well suited for this technique.
Figure 11 shows a calculated comparison of the ratio of these two gamma rays versus the factor required to correct for attenuation of the 414-keV gamma ray. An interesting feature is that the change in the 414$\mathrm{keV} / 129 \mathrm{keV}$ gamma ratio over the range of 0 to 100 grams plutonium per liter is almost entirely caused by the change in plutonium concentration; i.e., the ratio technique is quite insensitive to acid molarity. Matrix elements heavier than calcium (atomic number 20) can, however, affect the ratio sufficiently to jus. tify compensation with calibration standards of similar composition. With this in mind, we can use the ratio technique as the basis for a calibration curve as shown in Fig. 12.

Transmission or differential attenuation techniques such as those described here are necessary to obtain reliable measurements of plutonium concentration under static conditions. Under dynamic-flow conditions, however, the solution flow rate also is required for calculating the total quantity of plutonium that passes the measurement point. Quantitative measurement of dynamic flow appears to be the most challenging remaining problem. Because a continuously varying flow rate is particularly difficult to measure, the uncertainty of the flow rate measurement is expected to limit the reliability of any such measurement.

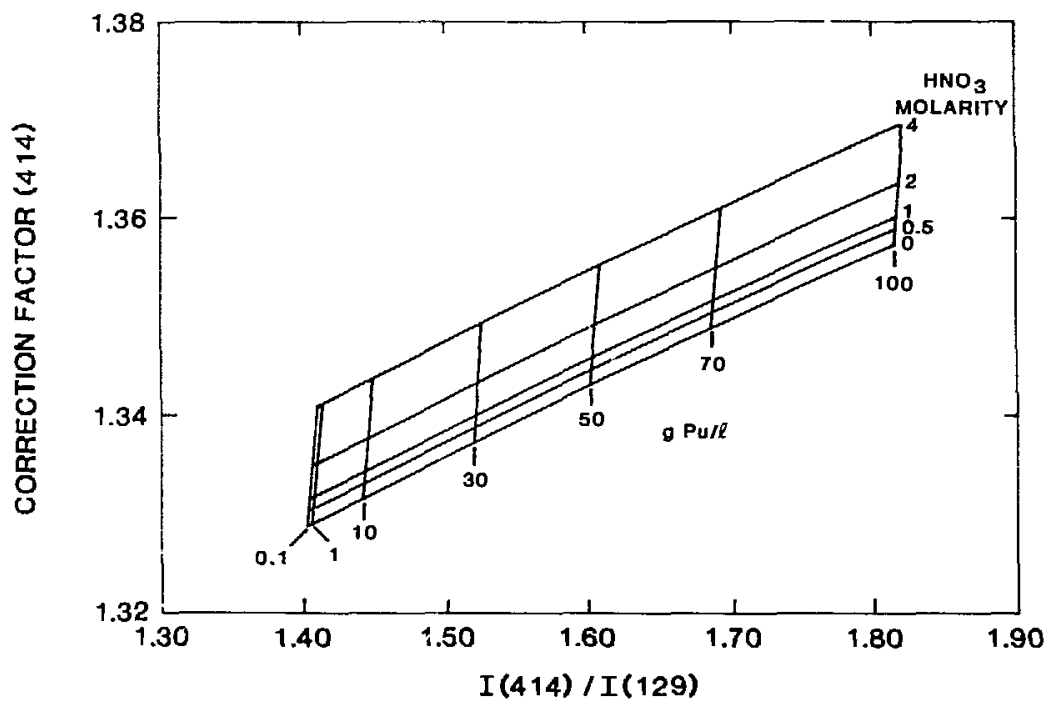

Fig. 11. Correction factor for attenuation of the $414-\mathrm{keV}$ gamma ray of ${ }^{239} \mathrm{Pu}$, based on the measured $414 \mathrm{keV} / 129 \mathrm{keV}$ intensity ratio. 


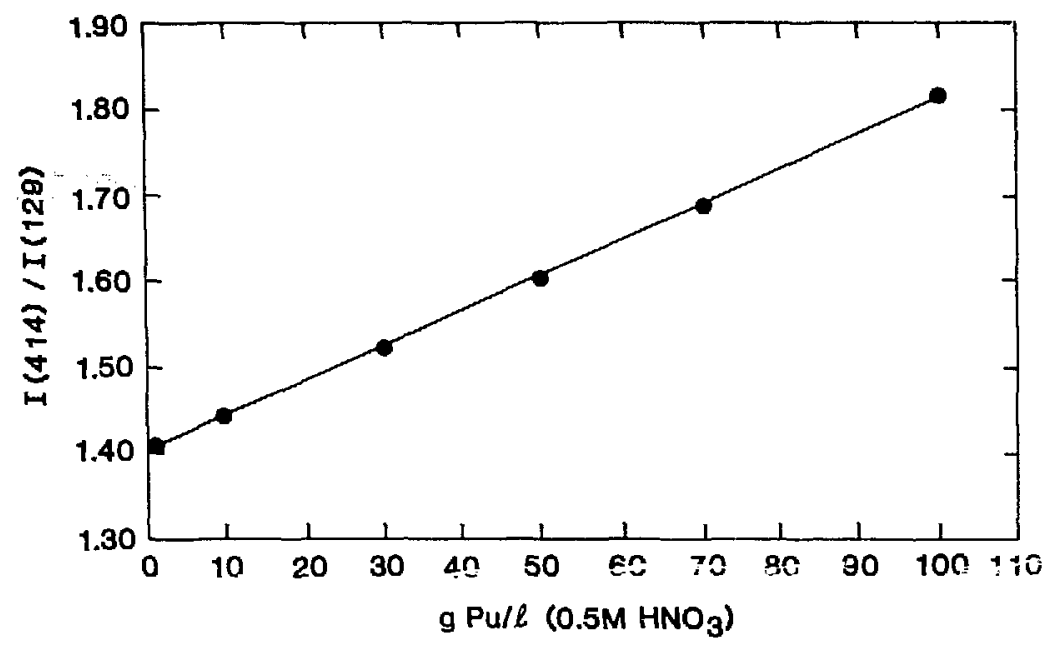

Flg. 13. Intensity ratios of $414-\mathrm{keV} / 129-\mathrm{keV}$ gamma rays as a function of plutonium concentration.

\section{REFERENCES}

1. S. F. Marsh and T. D. Gallegos, "Chemical Treatment of Plutonium with Hydrogen Peroxide Before Nitrate Anion Exchange Processing," Los Alamos National Laboratory report LA-10907 (May 1987).

2. S. F. Marsh and S.-T. Hsue, "Plutonium Process Control with an On-Line Gamma Monitor for Uranium, Plutonium, and Americium," Karlsruhe International Conference on Analytical Chemistry in Nuclear Technology, Karlsruhe, Federal Republic of Germany, June 3-6, 1985.
3. J. L. Parker, "The Use of Calibration Standards and the Correction for Sample Self-Attenuation in Gamma-Ray Nondestructive Assay," Los Alamos National Laboratory report LA-10045 (August 1984).

4. T. D. Reilly and J. L. Parker, "A Guide to Gamma-Ray Assay for Nuclear Material Accountability," Los Alamos Scientific Laboratory report LA-5794-M (March 1975). 